\title{
Activation of a Quiescent Choroidal Neovascularization in a Patient with Age-Related Macular Degeneration
}

\author{
Anna Matysik-Woźniak ${ }^{a} \quad$ Anat Loewenstein $^{\mathrm{b}} \quad$ Emilia Bielecka $^{\mathrm{a}}$ \\ Robert Rejdak ${ }^{a}$ \\ ${ }^{a}$ Chair and Department of General and Pediatric Ophthalmology, Medical University of \\ Lublin, Lublin, Poland; bDepartment of Ophthalmology, Tel Aviv Medical Center, Sackler \\ Faculty of Medicine, Tel Aviv University, Tel Aviv, Israel
}

\section{Keywords}

Age-related macular degeneration · Intravitreal injection - Quiescent choroidal neovascularization

\begin{abstract}
We report on a case of an unusual activation of one of 2 quiescent, nonexudative choroidal neovascular membranes in a patient treated with anti-VEGF injections for exudative age-related macular degeneration in his fellow eye. After almost 2 years of close observation, one of these membranes became exudative, and anti-VEGF therapy was started. This case illustrates the unpredictability of subclinical neovascularization and confirms the utility of optical coherence tomography angiography in the diagnosis and follow-up of these changes. Patients with an exudative age-related degeneration in one eye should have their fellow eyes monitored meticulously.
\end{abstract}

(C) 2021 The Author(s).

Published by S. Karger AG, Basel

\section{Introduction}

The quiescent, treatment-naive, nonexudative or subclinical choroidal neovascularization (CNV) is type $1 \mathrm{CNV}$, located under the flat detachment of the pigment epithelium. There is neither retinal fluid visible on optical coherence tomography nor leakage on fluorescein angiography. This type of CNV could be detected by indocyanine green angiography. Therefore, nonexudative CNVs were rarely discovered prior to the advent of optical coherence tomography angiography (OCTA) [1]. Only a wider introduction of OCTA into clinical practice makes it possible to conclude that quiescent CNVs are much more frequent 
than previously assumed. The prevalence of subclinical CNV in fellow eyes of patients with unilateral exudative age-related macular degeneration (AMD) has been reported to be between 6.25 and $27 \%$ [2-6]. Nonexudative CNVs are considered to be the precursors of exudative CNVs, and close monitoring is recommended to avoid visual loss. However, the natural history and biomarkers of activation of these changes are not well known. Unpredictability can make the management more difficult. The aim of this work is to present an unusual case of a conversion of a treatment-naive, nonexudative CNV to an exudative form. This case should raise the awareness in terms of proper diagnosis and careful follow-up of subclinical, quiescent CNVs.

\section{Case Presentation}

In 2015, a 59-year-old man was diagnosed with exudative AMD in his left eye. The diagnosis was based on fundus examination, optical coherence tomography, and fluorescein angiography. His best-corrected visual acuity (BCVA) in the left eye was 0.4. Since then, he was regularly treated with anti-VEGF injections (aflibercept, according to the label). So far, overall, he has received 26 injections into the left eye (every 2 months). The right eye did not show any signs and symptoms of wet AMD (BCVA $=1.0)$.

In 2017, with the advent of OCTA or angio-OCT, we were able to use this technique in all patients with AMD. The angio-OCT en face examination (Avanti with AngioVue OCTA; Optovue, Inc., Fremont, CA, USA) confirmed the presence of CNV in the left eye. Surprisingly, OCTA revealed the presence of 2 quiescent, asymptomatic, nonexudative type 1 CNVs in the right eye, too (shown in Fig. 1). The bigger CNV was irregular, glomerular-
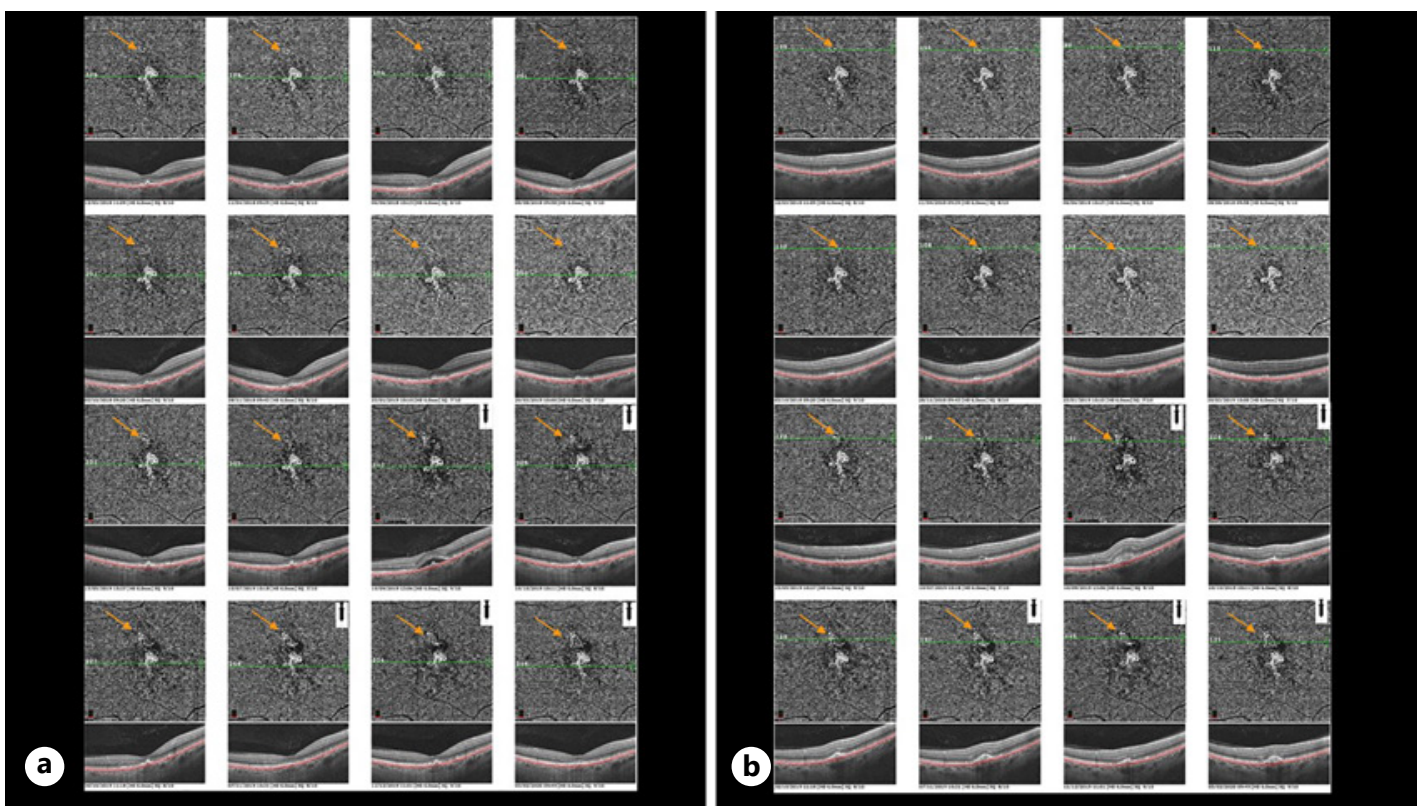

Fig. 1. OCT angiograms en face over a $6 \times 6-\mathrm{mm}$ area centered at the fovea (a slab between the outer retina and Bruch's membrane) with angio B-scans crossing the bigger CNV (a) and crossing the smaller CNV (b), showing the history of follow-ups of the right eye from 2018 to 2020 . The arrows point to the smaller CNV, which grows over time and becomes exudative. The symbol of the syringe indicates the time of the anti-VEGF injections (Avanti with AngioVue OCTA; Optovue, Inc., Fremont, CA, USA). CNV, choroidal neovascularization; OCTA, optical coherence tomography angiography. 
shaped, composed of small, rather dense, capillaries with longer tangled filaments located under the RPE, in the subfoveal region. The smaller one was more difficult to detect. It had more regular and round shaped with loops, located in the upper area of the macular region. Structural OCT showed flat, irregular pigment epithelium elevation in the areas corresponding to both CNVs; no fluid was observed, and BCVA was 1.0. Since then, we have performed the angio-OCT examination at least every 2 months. The shape and size of these nonexudative CNVs remained unchanged, and the patient did not report any disturbances in the right eye for almost 2 years. Then on May 2019, we observed the activation of the upper, smaller CNV, which started to grow, producing sprouting, dense and thin capillaries with loops, yet BCVA was still 1.0. However, the growth of the pigment epithelial detachment was not pronounced. Four months later, subretinal and intraretinal fluid, subretinal hyperreflective mass, retinal hemorrhages, and perilesional dark halo appeared, and BCVA decreased to 0.7 (shown in Fig. 2). The shape, size, and morphology of the bigger, subfoveal CNV seemed to be the same. The anti-VEGF therapy was started immediately, and the patient responded well to the treatment (so far, he has received 5

Fig. 2. a OCT angiograms en face over a 3 $\times 3-\mathrm{mm}$ area centered at the fovea (a slab between the outer retina and Bruch's membrane) with angio B-scans crossing the bigger CNV (left) and the smaller CNV (right), showing exudation and hyperreflective mass as well as a shallow RPE detachment. At the moment of activation (Avanti with AngioVue OCTA; Optovue, Inc., Fremont, CA, USA). b Fundus photography showing intraretinal hemorrhages corresponding to the activated, smaller CNV (Topcon 3D OCT-2000). CNV, choroidal neovascularization; OCTA, optical coherence tomography angiography.
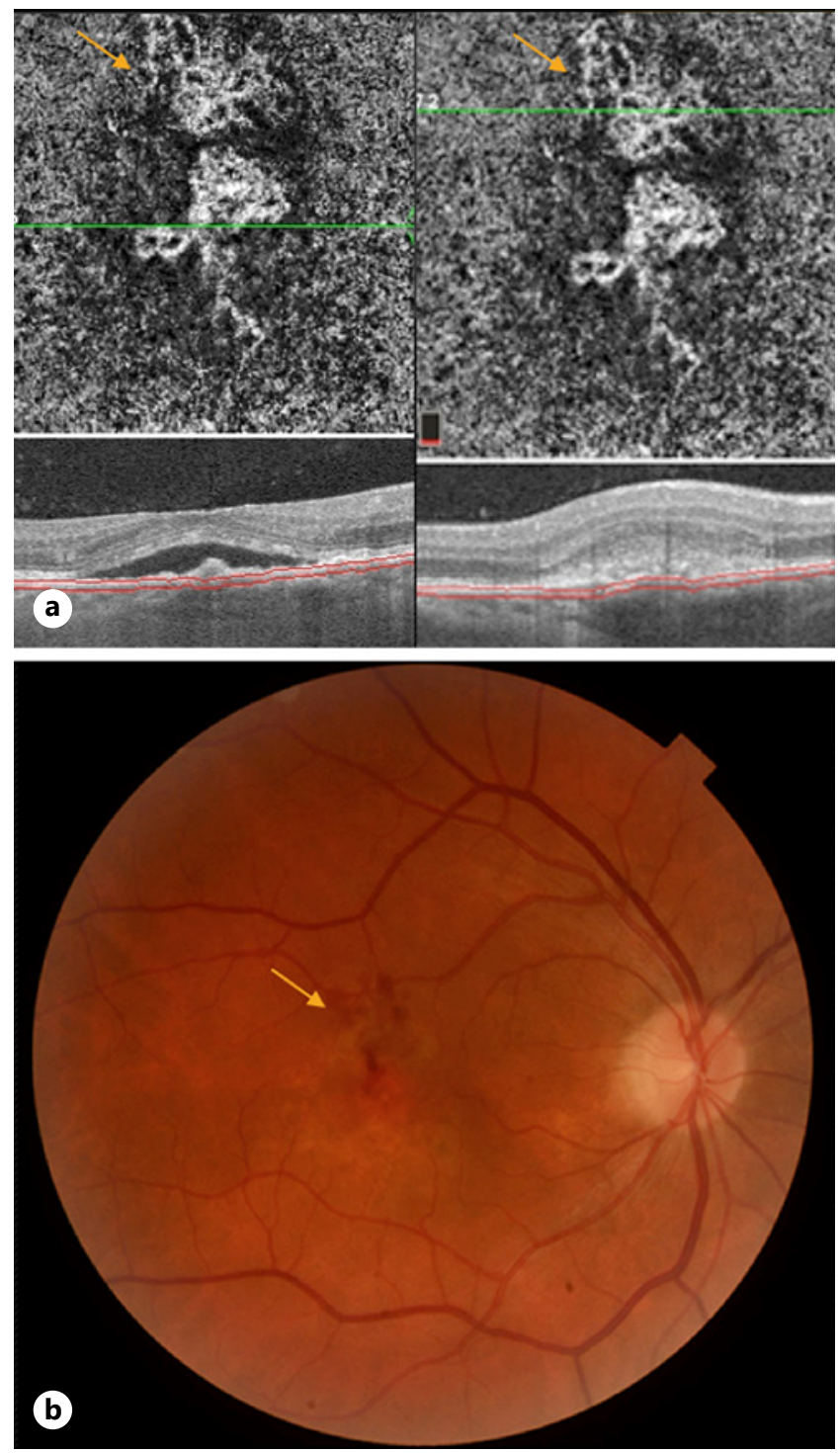
Matysik-Woźniak et al.: Activation of Choroidal Neovascularization

injections of aflibercept according to the label). His BCVA improved to 1.0. The edema has gone; the subretinal fluid and retinal hemorrhages have disappeared, but the shape and size of the CNVs have not changed.

\section{Discussion}

Nonexudative CNVs are composed of more mature vessels with competent endothelial cell junctions, which prevents exudation. They could be segregated as previously treated with anti-VEGF injections or treatment-naive CNVs. The former ones are the result of type $1 \mathrm{CNV}$ evolution and arterialization or constitute residual blood channels in fibrotic scars. Their vessels are rigid, thicker, and straighter lacking thinner capillaries. However, some of them may require intermittent injections to prevent growth and exudation [1]. Treatment-naive CNVs form tangled networks or filament-like structures and do not need treatment. Nevertheless, they may progress to exudation and growth. The exact relationship between exudative and nonexudative CNVs remains unknown [1].

We report on a case of an unusual activation of one of 2 quiescent, nonexudative CNVs in a patient treated with anti-VEGF injections for exudative AMD in his fellow eye. After almost 2 years of close observation, the smaller CNV became exudative, and anti-VEGF treatment was started. The exudation was preceded by the enlargement of the smaller CNV area, while the bigger one seemed to be unchanged. The presence of intraretinal hemorrhages and hyperreflecitve mass above the RPE may suggest that the smaller CNV developed a part which was CNV type 2. It responded early and well to anti-VEGF therapy.

It is difficult to explain why only one smaller CNV started to grow and became active. It could be speculated whether the morphology and shape could play a role. However, in the described case, both CNVs had irregular shapes and were small, tangled vessels with loops. It seemed that the smaller CNV had a less dense structure. Initially, the dark halo was more pronounced around the bigger CNV. According to Lumbroso et al. [1], the shape of nonexudative CNVs is mostly irregular and rounded in $20 \%$. Most membranes are large networks with large vessels and had very few or no small capillaries. Loops and halo are occasional [1].

It has been reported that eyes with subclinical CNV at the time of the first OCTA imaging had a 15-fold higher risk of exudation developing than eyes without detectable MNV [3]. According to another observation of 10 quiescent, treatment-naive CNVs, $80 \%$ of them begin to exudate within 10 months. This was preceded by a rapid growth in the lesion size, as measured by OCTA [5]. We observed the enlargement of the smaller CNV and surrounding halo before exudation, after almost 2 years of follow-up.

Another interesting issue raised by many authors is whether an anti-VEGF therapy in the fellow eye can inhibit the progression and activity of quiescent CNV [1]. In our patient, the long and systematic anti-VEGF therapy in the fellow eye did not prevent the growth and exudation of the quiescent $\mathrm{CNV}$.

\section{Conclusions}

The presented case confirms the importance of careful examination of the fellow eye in patients diagnosed with wet AMD in one eye. It also shows that subclinical CNVs may be unpredictable precursors for exudative AMD. Vigilance is necessary for eyes with the quiescent, treatment-naive, nonexudative or subclinical CNVs. We recommend that these changes be observed at least every 2 months. The anti-VEGF injections are necessary, when

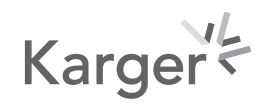


the signs of exudation appear. Future studies are needed to understand better the natural history of quiescent CNVs and useful biomarkers of its activation. OCTA is a very useful method for diagnosing and monitoring of subclinical CNVs.

\section{Statement of Ethics}

This work was performed according to the guidelines for human studies and was performed ethically in accordance with the World Medical Association Declaration of Helsinki. Prior written informed consent was obtained from the patient. This report does not contain any personal information that could lead to the identification of the patient.

\section{Conflict of Interest Statement}

The authors declare no conflicts of interest.

\section{Funding Sources}

The authors did not receive any funding.

\section{Author Contributions}

A.M.-W.: writing and reviewing. A.L.: writing and reviewing. E.B.: writing and reviewing. R.R.: writing and reviewing.

\section{References}

1 Lumbroso B, Huang D, Souied E, Savastano M, Jia Y, Rispoli M Understanding OCT angiography from patophysiology to clinical imaging. London; New Dehli: Jeypee Brothers Mrdical Publishers; 2020. p. 85-90.

2 Heiferman MJ, Fawzi AA. Progression of subclinical choroidal neovascularization in age-related macular degeneration. PLoS One. 2019;14(6):e0217805-9.

3 de Oliveira Dias JR, Zhang Q, Garcia JMB, Zheng F, Motulsky EH, Roisman L, et al. Natural history of subclinical neovascularization in nonexudative age-related macular degeneration using swept-source OCT angiography. Ophthalmology. 2018;125(2):255-66.

4 Roisman L, Zhang Q, Wang RK, Gregori G, Zhang A, Chen C-L, et al. Optical coherence tomography angiography of asymptomatic neovascularization in intermediate age-related macular degeneration. Ophthalmology. 2016;123(6):1309-19.

5 Palejwala NV, Jia Y, Gao SS, Liu L, Flaxel CJ, Hwang TS, et al. Detection of nonexudative choroidal neovascularization in age-related macular degeneration with optical coherence tomography angiography. Retina. 2015; 35(11):2204-11.

6 Yanagi Y, Mohla A, Lee W-K, Lee SY, Mathur R, Chan CM, et al. Prevalence and risk factors for nonexudative neovascularization in fellow eyes of patients with unilateral age-related macular degeneration and polypoidal choroidal vasculopathy. Invest Ophthalmol Vis Sci. 2017;58(9):3488-95. 